\title{
Comparative gene expression between two yeast species
}

Yuanfang Guan ${ }^{1,2,3}$, Maitreya J Dunham ${ }^{1,4^{*}}$, Olga G Troyanskaya ${ }^{1,5^{*}}$ and Amy A Caudy ${ }^{1,6^{*}}$

\begin{abstract}
Background: Comparative genomics brings insight into sequence evolution, but even more may be learned by coupling sequence analyses with experimental tests of gene function and regulation. However, the reliability of such comparisons is often limited by biased sampling of expression conditions and incomplete knowledge of gene functions across species. To address these challenges, we previously systematically generated expression profiles in Saccharomyces bayanus to maximize functional coverage as compared to an existing Saccharomyces cerevisiae data repository.

Results: In this paper, we take advantage of these two data repositories to compare patterns of ortholog expression in a wide variety of conditions. First, we developed a scalable metric for expression divergence that enabled us to detect a significant correlation between sequence and expression conservation on the global level, which previous smaller-scale expression studies failed to detect. Despite this global conservation trend, between-species gene expression neighborhoods were less well-conserved than within-species comparisons across different environmental perturbations, and approximately $4 \%$ of orthologs exhibited a significant change in co-expression partners. Furthermore, our analysis of matched perturbations collected in both species (such as diauxic shift and cell cycle synchrony) demonstrated that approximately a quarter of orthologs exhibit condition-specific expression pattern differences.

Conclusions: Taken together, these analyses provide a global view of gene expression patterns between two species, both in terms of the conditions and timing of a gene's expression as well as co-expression partners. Our results provide testable hypotheses that will direct future experiments to determine how these changes may be specified in the genome.
\end{abstract}

Keywords: Expression divergence, Yeast, Microarray, Data integration, Condition-specific gene expression

\section{Background}

The Ascomycete yeasts present one of the most promising systems for comparative functional genomics. Fungi have been densely sampled by a number of sequencing projects [1], covering an enormous range of divergence. Genome sequence analyses of the Saccharomyces yeasts and related species have been used to establish the history of gene duplication [2-6], conservation at binding sites $[7,8]$, and co-evolution of binding sites with

\footnotetext{
* Correspondence: maitreya@uw.edu; ogt@cs.princeton.edu; amy.caudy@ utoronto.ca

${ }^{1}$ Lewis-Sigler Institute for Integrative Genomics, Princeton University, Princeton, NJ 08544, USA

${ }^{4}$ Department of Genome Sciences, University of Washington, Seattle, WA 98195, USA

Full list of author information is available at the end of the article
}

regulators [9]. Thus, a range of evolutionary phenomena can be studied in these species based on their genomic sequence. However, sequence conservation is not always completely predictive of functional conservation. As just one example, we recently reported that only a subset of conserved promoter motifs actually drive periodic gene expression over the cell cycle in two closely related species [10].

Most of the experimental characterization of gene function has been performed in a small number of model fungal systems, which can provide an anchor for these broad genome sequencing surveys. These species include Saccharomyces cerevisiae, Neurospora crassa, Candida albicans, and Schizosaccharomyces pombe, along with several other emerging models such as Ashbya gossypii. Comparative studies between these species, which 
by some estimates cover a billion years of divergence, have been informative [11,12]. Analysis of gene expression changes over growth [13], the cell cycle [13], and stress treatments $[14,15]$ highlighted both similarities and differences in ortholog expression. Unfortunately, the ability to link individual gene expression divergence with the causative molecular factors has been limited because of the vast evolutionary distances involved.

Experimental protocols developed in the model systems are often readily portable to less well-studied sister species, allowing us to choose species well-placed to identify and study functional divergence. Comparisons of gene expression across particular species with interesting characteristics can not only highlight how patterns of gene expression change over evolutionary time, but can also discover genes with particular functions. A comparison among xylose-metabolizing species of yeasts, for example, was able to couple sequence analysis with gene expression profiling to identify important genes via their presence in the genomes of interest and their induction when grown on xylose [16]. Followup studies in S. cerevisiae confirmed these associations.

Due to their close proximity to $S$. cerevisiae, studies in the sensu stricto yeasts have also been particularly informative. These species cover a range of conservation, have high quality annotated reference genomes [17], and are becoming even more attractive as the sequences of many strains within each species are forthcoming using new high-throughput sequencing tools (e.g. [18]). Furthermore, their ability to form interspecific hybrids leverages the resources available in S. cerevisiae and allows tests of gene function and regulation in shared cellular environments [19-23]. Recent work on expression-based full-genome characterization is reported in $[24,25]$, which used $S$. cerevisiae microarrays to measure the gene expression consequences of heat shock stress and mating induction on three other yeast species. Their data suggest that expression divergence can occur relatively rapidly and is correlated to gene function, though relatively uncorrelated to sequence conservation [26]. Due to the $S$. cerevisiae arrays used, they were unable to examine more divergent species. In order to broaden these studies to more divergent yeasts, species-specific arrays must be used, as has been done, for example, for Candida glabrata [27]. Most importantly, due to the limited condition space of just a small number of treatments in these studies, conclusions about evolution of gene function and regulation have been difficult to generalize.

To address these challenges, we previously developed a computational framework $[28,29]$ to identify a set of experiments that could best characterize gene function in a naive species. Based on available expression date in the $S$. cerevisiae literature, we identified and carried out a set of 304 experiments over 46 conditions in the sensu stricto species $S$. bayanus var uvarum. By choosing only the most informative experiments from the vast $S$. cerevisiae literature, we were able to survey a large phenotypic space at high accuracy with a modest amount of experimentation.

To compare these expression datasets more carefully, we developed a statistical metric, Local Network Similarity (LNS), to assess correlation patterns of orthologs. This metric is general and robust - it can be used for analysis of individual matched datasets without the need to assume identical response time for the two species, or for integrated analysis of diverse compendia of experimental or genetic perturbations. Using the LNS metric to compare our large S. bayanus expression compendium with a collection of published $S$. cerevisiae expression data, we show that gene expression networks are largely conserved between the species, though much less than within-species comparisons constructed by comparing different conditions. Furthermore, we demonstrated strong and statistically significant evidence for correlation between the divergence of expression and open reading frame sequence, which previous studies using more limited datasets failed to detect (see review [26]). Despite this general conservation pattern, we observed that a quarter of orthologs exhibit conditionspecific differences in expression, and $4 \%$ show strong differences in global co-expression patterns. Genes involved in the same functional groups share similar divergence patterns, indicating that pathways or processes may share characteristics. In sum, our wide-ranging survey of expression profiles and generic metric of expression divergence allowed us to identify both global and local aspects of regulatory evolution and relate these to sequence divergence.

\section{Results}

S. bayanus var uvarum (referred to as "S. bayanus" from here forward) is a sequenced but relatively unstudied species of budding yeast typically associated with fermentation environments and diverged by approximately 20 million years from $S$. cerevisiae. We have recently computationally chosen 46 biological treatments for gene expression analysis that would maximally cover biological processes in yeast [28]. Using the resulting dataset of over 300 arrays in S. bayanus along with 2569 arrays collected from S. cerevisiae [30-34], we carried out both global and dataset-specific comparisons of gene expression.

\section{A generic statistical metric to quantify expression conservation between species}

To measure the divergence in gene expression between the two species, we developed a metric to compare the expression networks surrounding orthologous genes. Since gene co-expression is strongly linked to functional 
similarities, differences in expression neighborhoods over a reasonably comprehensive set of perturbations provide a robust proxy of functional relationships. Previously developed methods of analyzing co-expression patterns across species have relied on producing matched datasets, in which comparable timepoints were collected from multiple species exposed to the same conditions [24]. However, exactly matched datasets are difficult to gather, and such an approach relies on the assumption that the precise timing of expression change should be conserved. We sought to develop a method that could detect large-scale patterns of co-expression in addition to those found under specifically matched conditions.

In order to quantify expression divergence at this global level, we developed a metric, Local Network Similarity (LNS), which measures the similarity of expression connections around each member of an ortholog pair (Figure 1). This metric is conceptually inspired by previous analyses that summarize the entire network of coexpression that exists between a gene and the rest of the genes in the genome $[35,36]$. The distribution of correlation coefficients of different datasets varies greatly, as is demonstrated in Figure 1, which plots actual expression datasets from the two species. LNS is calculated by stabilizing the variance of the distribution of correlation coefficients by first normalizing the within-species genegene correlation coefficients using the Fisher transformation and then further normalizing these data to the standard normal distribution. This normalization prevents domination by few changes of large magnitude or loss of signal from changes that occur in only a subset of perturbations (see Methods for details). The expression conservation of a pair of orthologous genes is thus quantified based on the preservation of all the expression connections around the pair of orthologs, i.e., the similarity of the local, first-degree expression networks around the two genes. The distribution of LNS of a completely non-conserved network resembles the normal distribution, making direct hypothesis tests possible.

Notably, and in contrast to previous global comparison approaches, this definition does not rely on alignment of individual datasets but defines a gene's expression pattern in the context of the genome-wide co-expression network. Therefore, the LNS concept could be extended to integrate any number of genome-scale expression datasets-or even other types of genomic data-to quantify conservation between any two species pairs.

\section{Global expression conservation and divergence identified by LNS}

LNS revealed considerable conservation of correlation structure between the two species' expression networks. We simulated the case of zero conservation by ran- domizing the orthologous pairs while preserving the network structure (Figure 1). This simulation resulted in LNS scores normally distributed and centered at 0 (Figure 2A). Unlike the randomized network, the LNS scores based on the matched orthologs were distributed from -0.63 to 0.83 with the median of 0.45 (Figure 2A, Additional file 1: Table S1), showing a clear shift towards positive values. This demonstrates that on average, orthologs preserved their expression network. A right shoulder in the LNS distribution suggests that there is a subset of genes with very highly conserved coexpression networks; this population of genes with high LNS persists even when ribosomal genes are removed from the data (data not shown).

In order to test whether this conservation is more or less than what would be expected at this degree of sequence divergence, we would ideally compare a similar gene expression compendium collected from genetically diverse isolates within each species or in species at different genetic distances. Although some data exist exploring differences in gene expression among $S$. cerevisiae strains [37-39], there are not sufficient datasets available for a full test. However, we were able to test the limit case of LNS as compared between different subsets of the $S$. cerevisiae literature. These experiments survey a wide diversity of conditions and have been performed in different strain backgrounds, though mostly focused on a small number of related lab strains. The LNS distribution within the subsampled $S$. cerevisiae datasets was significantly more positive than both the random distribution and the between species comparison (Figure 2A, Additional file 2: Table S2). This result demonstrates the robustness of LNS for identifying patterns of co-expression even across very different environmental and genetic conditions, and suggests that the level of coexpression difference observed between $S$. cerevisiae and $S$. bayanus is probably beyond what is present within a species.

Cross-species LNS revealed some dramatic changes in correlation patterns in addition to the overall pattern of conservation. We found 183 genes out of the 4701 orthologous pairs (4\%) with an LNS lower than 0, suggesting these genes changed globally in their expression network (Additional file 1: Table S1). The orthologs with low LNS represent several underlying biological causes. One obvious category is genes known to carry deficiencies in laboratory strains. For example, the promoter of CTR3 is disrupted by a Ty2 insertion in many lab strains of $S$. cerevisiae [40], but is intact in S. bayanus. Most of the data in the S. cerevisiae compendium is derived from lab strains in which CTR3 expression is driven by the transposon promoter, while the native promoter is present in the $S$. bayanus strains used in our compendium, leading to very different expression patterns and a low LNS score of -0.07166 . 


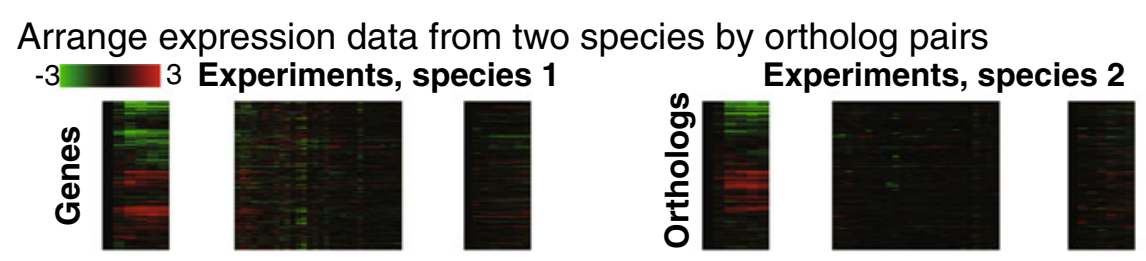

Calculate Pearson correlation of all gene-gene pairs within each species

Distribution of correlation values
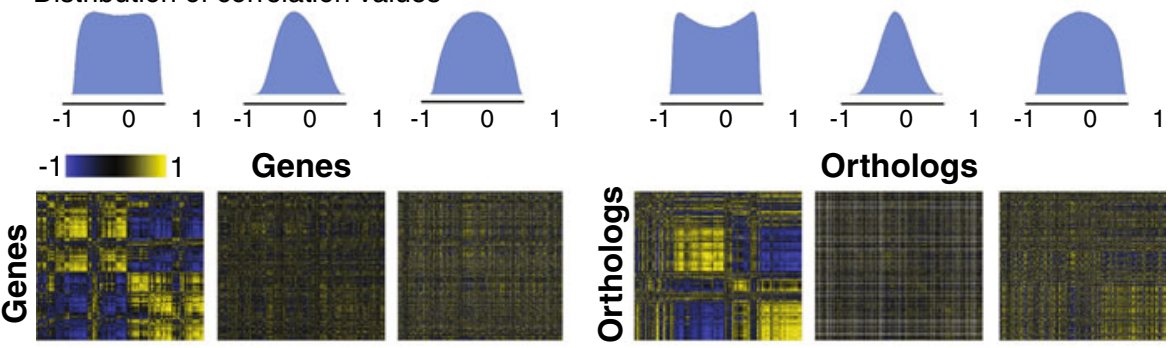

Rescale correlation matrix by Z-transformation

Distribution of z-transformed correlation values
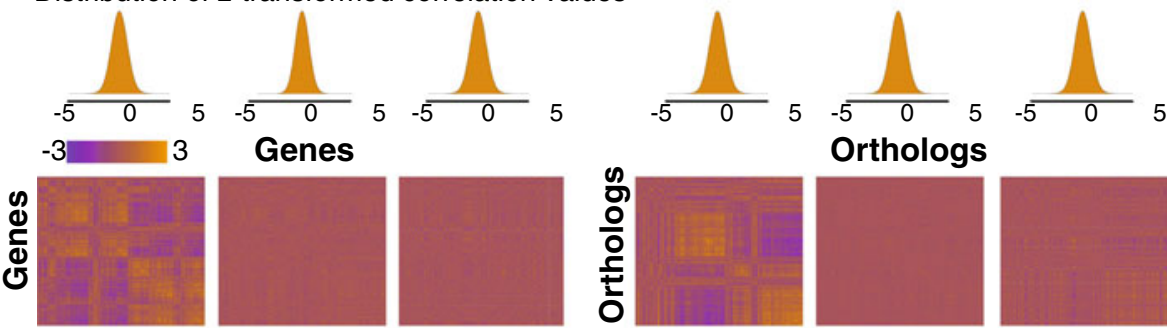

Average Z-transformed correlation matricies within species

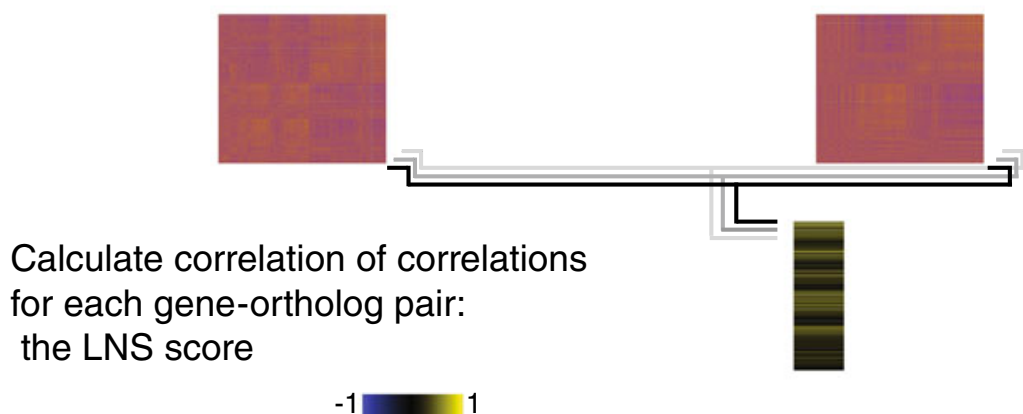

Figure 1 Quantification of expression conservation by local network similarity (LNS). Pair-wise Pearson correlation between genes was calculated for individual S. bayanus and S. cerevisiae datasets, generating a matrix of gene-gene correlations. The data used to create this illustration are the actual diauxic shift, cell cycle synchronization, and alpha factor treatment. The distribution of these correlation values is between -1 and 1, and can be drastically different from one dataset to another. Therefore, Fisher's z-transformation and normalization of these zvalues were applied on each correlation matrix, so that the correlations were comparable across datasets. The resulting correlation matrices are normally distributed and centered at 0 with standard deviation equal to 1. For each orthologous pair $i$ and $i$,', their $z$-transformed, normalized correlation to all other matched orthologs form two vectors, indicating the relative positions of this pair of ortholog in their respective expression network. The correlation of these two vectors was taken as LNS. To calculate the correlation matrix for global LNS, the average values of individual datasets for a specific gene-gene pair were used to form a new global correlation matrix. According to the properties of normal distribution, the values within this matrix are still normally distributed and centered at 0 with standard deviation equal to 1. This global matrix was then used to calculate global LNS for each ortholog. To simulate the case of non-conservation, orthologous pairs were randomized along one axis of the expression correlation matrix. Therefore in calculating background LNS, only the ortholog match was disturbed, but not the expression network structure (in contrast to randomizing along both axes). 


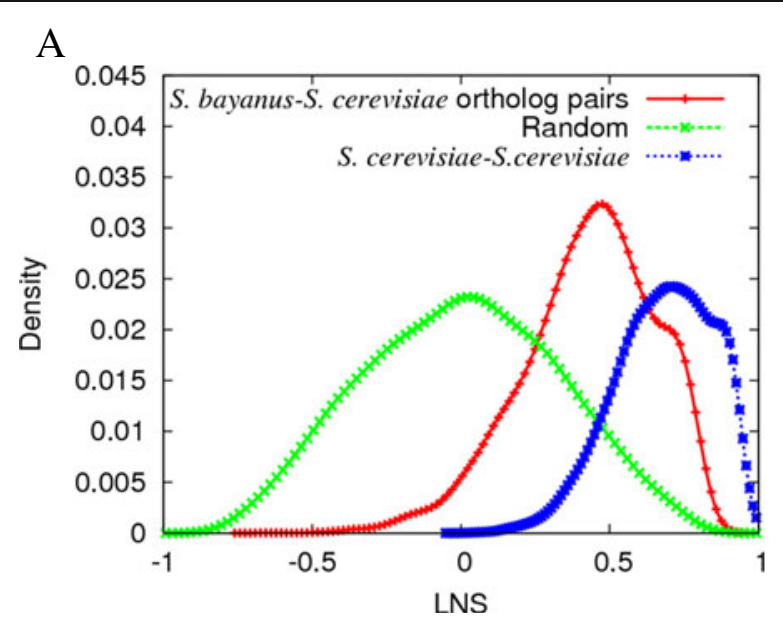

B

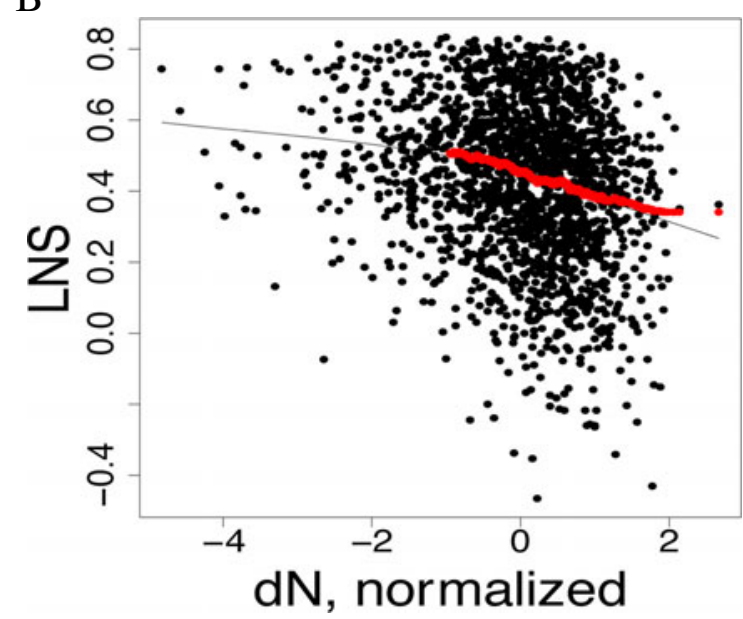

Figure 2 Expression conservation relationship with sequence conservation. A. Compared to randomized gene pairs, distribution of LNS scores of all one-to-one ortholog pairs is shifted towards positive values, though this distribution is less positive than comparisons within species. B. LNS is correlated with sequence divergence with a Pearson correlation of -0.235 ( $p<2.2 e-16)$. Black line is loess smoothed curve line and red line is running average of LNS summed over 100-gene windows. Graphs for other measures of sequence divergence are in Figure S2.

Similarly, we noted a number of targets of alpha factor signaling among the lowest LNS scores, and subsequently examined a list of known transcriptional targets of signaling through Gpa1, the alpha component of the heterotrimeric $\mathrm{G}$ protein that activates the MAP kinase cascade in yeast [41]. The median LNS of the GPA1 targets is 0.27 , significantly lower than the set of all genes. S288c derived laboratory strains of S. cerevisiae carry a S469I GPA1 mutation that increases signaling through the MAP kinase cascade, but S. bayanus and all other members of the sensu stricto group carry the ancestral allele [42]. Therefore, the low overall LNS scores of the GPA1 target genes may reflect the difference between wild type GPA1 activity in S. bayanus and hyperactive signaling in laboratory strains of $S$. cerevisiae. Background mutations in $S$. bayanus can be identified as well, including the alpha factor protease gene [43], $B A R 1$, which is mutant in the $S$. bayanus strain used in nearly every experiment in our expression compendium, and scored a low 0.26 LNS.

The second category of low LNS scores represents incorrectly annotated ortholog pairs. In our initial analysis, we discovered a number of cases of improperly assigned orthologs (Table 1). For instance, the S. bayanus gene 620.38 had been assigned the ortholog RAS1 during the initial annotation effort [8], and had an LNS of -0.38 . However, the protein sequence of the 620.38 ORF has only partial homology to Ras1 and is in fact a close homolog of the vacuolar protein Vps21. In addition, 620.38 is syntenic with VPS21 [44]. This example demonstrates that LNS provides a functional criterion for ortholog identification and validation.

The remaining genes with low LNS are from diverse biological processes and functions. These genes are enriched for orthologs whose S. cerevisiae annotations include genes involved in ascospore cell wall formation (GO:0009272 fungal cell wall-type biogenesis, Bonferroni-corrected p-value $8.97 \times 10^{\wedge-5}$ and related terms), and other developmental processes involved in mating and meiotic division, leading to the intriguing hypothesis that gene expression network differences may be related to speciation between these two yeasts. However, genes associated with these biological processes accounted for only $15 \%$ of the highly divergent genes. Multiple genes in this set are associated with DNA synthesis and repair, signaling, chromatin organization, metabolism, and transcription, among many other processes, emphasizing that differences are present throughout the cellular network. This list of low LNS genes in known functions should assist the prioritization of experimental work to determine the basis of evolutionary changes in expression. A full quarter of the genes with low LNS scores are of unknown function. Further experiments will be required to determine the mechanisms by which these genes diverge in their expression networks, and the degree to which these differences may contribute to phenotypic differences between the species.

\section{Sequence conservation predicts gene expression divergence}

Despite the requirement for experimental followup of individual ortholog pairs, LNS analysis on our large data collection allows us to test several hypotheses regarding the overall role of genome sequence in determining interspecies variation of gene expression. First, we considered the effect of promoter structure by grouping ortholog pairs into TATA-containing in both species, TATA-containing in one of the members, and TATA- 
Table 1 Mis-annotated genes identified by LNS

\begin{tabular}{|c|c|c|c|c|}
\hline Gene & Old ortholog & $\begin{array}{l}\text { New } \\
\text { ortholog }\end{array}$ & LNS & Comments \\
\hline 620.38 & YOR101W & YOR089C & -0.379 & Blast e-value to VPS21 is $1 \mathrm{e}-92$. Blast to RAS1 is 11 th on list. \\
\hline 576.11 & YGL157W & YGL039W & 0.184 & $\begin{array}{l}\text { Note similar synteny conflict with } 674.45 \text {. This gene is second best blast, e-value 1e- } \\
160 .\end{array}$ \\
\hline 674.45 & YGL039W & YGL157W & -0.077 & $\begin{array}{l}\text { Synteny preserved by change, new gene is best blast hit with e-value } 3 e-146 \text {. Note } \\
\text { similar problem with } 576.11 \text {. }\end{array}$ \\
\hline 635.17 & YOR267C & YOR233W & -0.633 & Synteny preserved by change. YOR233W is best blast hit with e-value 0 . \\
\hline 636.13 & YPR119W & YPR120C & -0.03 & \\
\hline $\begin{array}{l}\text { (cell } \\
\text { cycle) }\end{array}$ & $\begin{array}{l}\text { Best hit with CLB5 } \\
\text { (YPR120C). }\end{array}$ & & & \\
\hline
\end{tabular}

Ortholog pairings were initially taken from the original genome annotation [7]. Several mis-pairs were identified by their low global or condition-specific LNS.

less. Though not statistically significant (Additional file 3: Figure S1, $r=-0.02, p=0.08$ ), TATA-containing orthologs have lower LNS, indicating higher interspecies variation, consistent with previous results [24] using other yeast species and measurements of expression divergence.

Secondly, changes in promoter sequence could potentially cause changes in gene expression, so we extended the evaluation of promoters to examine the relationship between upstream sequence conservation and local network similarity. Upstream sequence conservation is weakly predictive of expression conservation (Additional file 3: Figure S1, $r=0.047, p=0.00016, n=4701$ ).

Thirdly, in contrast to the small effects of TATA promoter type or upstream sequence conservation, we found a stronger correlation (Figure 2B, $r=-0.235, p<$ $2.2 \mathrm{e}-16)$ between protein sequence similarity and local network similarity. This relationship was observed when using several different methods of calculating sequence similarity (Additional file 3: Figure S2). This correlation between protein sequence and expression similarity is consistent with the majority of results in mammalian systems [45-47], Хenopus [48] and Drosophila [49,50]. This result contrasts with the conclusions of previous studies in yeast that did not detect any relationship between sequence conservation and expression conservation [26]. Our ability to detect this relationship may result from our use of a large, diverse expression compendium and a more generic measurement of expression divergence. Indeed, if we focus solely on a pair of cell cycle datasets and align them by time points, similar to previous works $[24,25]$, we do not detect correlation between sequence conservation and expression conservation (not shown). As a result, although using aligned datasets could help identify orthologs responding with a similar pattern to a particular biological perturbation, calculation of expression correlation of orthologs in a single dataset cannot satisfactorily represent the expression conservation level.

\section{Major condition-specific changes in expression between S. bayanus and S. cerevisiae}

Using an expression compendium, the global LNS measures general expression change between species but may not be sensitive to changes in condition-specific expression patterns in response to specific environmental or genetic perturbations. For instance, a gene may be expressed at a basal level in one condition, but be strongly activated relative to other genes in a second condition. For example, the effect of divergence of Ste12 binding sites on alpha factor gene expression response was only detected in an alpha factor dataset and not in a larger expression compendium [25], likely because there is minimal alpha factor signaling under conditions where alpha factor is absent. Since we anticipate that genes highly conserved in expression response in one condition could diverge significantly in another condition, so we investigated expression conservation using a datasetspecific approach. In order to more sensitively examine condition-specific gene expression conservation, we calculated the LNS for individual datasets or small, related groups of datasets similar in size and perturbation and examined the behavior of different functional groups (Table 2). This measure, which we call condition-specific LNS (Additional file 4: Table S3), is still independent of the timing of perturbation response in the two species, but provides a condition-specific measure of expression divergence, enabling us to more sensitively detect divergence that is specific to a particular condition.

We found that, overall, orthologs exhibited some level of conservation in expression pattern regardless of the treatment, as expressed in the bias towards positive condition-specific LNS across matched datasets (Figure 3 and Table 2). However, individual experimental treatments and genetic perturbations demonstrated different patterns of expression conservation. For example, in the heat shock and diauxic shift treatments, genes on average showed high expression conservation (Figure 3 and Table 2). Other perturbations, for example, alpha factor 
Table 2 Paired datasets of S. bayanus and S. cerevisiae for condition-specific LNS calculation

\begin{tabular}{|c|c|c|c|c|}
\hline $\begin{array}{l}\text { S. bayanus } \\
\text { dataset }\end{array}$ & $\begin{array}{l}\text { S. cerevisiae } \\
\text { dataset }\end{array}$ & $\begin{array}{l}\text { Number of diverged genes (lower than average of randomization, } \\
\text { cutoff) }\end{array}$ & $P$-value & $\begin{array}{l}\text { Median } \\
\text { LNS }\end{array}$ \\
\hline MMS & [51] & $1498(-0.02515)$ & $1.67 \mathrm{E}-153$ & 0.2348 \\
\hline Heat shock & [52] & $1032(-0.00467)$ & $4.25 \mathrm{E}-271$ & 0.2259 \\
\hline Sorbitol & [52] & $1330(0.00561)$ & $3.35 E-163$ & 0.1678 \\
\hline Uracil & [53] & $1590(0.001)$ & $\begin{array}{l}5.75443 E- \\
82\end{array}$ & 0.1031 \\
\hline Rapamycin & [54] & $1896(-0.0101)$ & $\begin{array}{l}1.63545 \mathrm{E}- \\
33\end{array}$ & 0.07464 \\
\hline Alpha factor & {$[55]$} & $1438(-0.006)$ & $\begin{array}{l}5.5186 \mathrm{E}- \\
102\end{array}$ & 0.0848 \\
\hline Sporulation & {$[56]$} & $922(0.0038)$ & $\begin{array}{l}3.7792 \mathrm{E}- \\
288\end{array}$ & 0.08923 \\
\hline Cell cycle & {$[57]$} & $1124(0.0047)$ & $\begin{array}{l}1.7278 \mathrm{E}- \\
226\end{array}$ & 0.09546 \\
\hline Diauxic shift & [58] & $947(0.00656)$ & $\begin{array}{l}1.1322 \mathrm{E}- \\
257\end{array}$ & 0.3741 \\
\hline
\end{tabular}

We permuted gene-gene correlations to simulate the LNS distribution of a randomized network. The numbers of genes below the average LNS for these random networks were counted. We also calculated the $p$-value by randomizing the matches between orthologs to simulate non-conservation. The average LNS for each matched dataset pair was calculated.

treatment, uracil limitation, and rapamycin treatment, exhibited a relatively higher number of genes with divergent expression, although the majority of genes were still well-conserved in these datasets. The differences among the LNS distributions are most likely due to both the properties of the experimental treatment and the quality and size of the two datasets compared for each treatment. In order to normalize these effects and attempt to quantify the number of genes with divergent expression across matched datasets, we randomized the ortholog match for each dataset pair so as to simulate the situation of no conservation. In other words, to generate the randomized set, for all ortholog pairs, one member of the pair was matched with a random ortholog in the other species (with the random ortholog coming from some other orthologous pair). In general, around a quarter of the orthologs had a condition-specific LNS lower than the average LNS of randomized datasets. This indicates even very conserved biological responses elicit different gene expression consequences in the two species.

Genes of different functional categories showed differential levels of expression divergence under specific conditions. In general, genes of ribonucleoprotein complex biogenesis and assembly (a term which contains primarily genes involved in ribosome structure and assembly) showed highly conserved expression patterns regardless of the nature of the expression perturbation. Other categories of genes showed more specific patterns of conservation. For example, cell cycle genes were most conserved in the cell cycle dataset and alpha factor treatment. In datasets such as rapamycin or uracil treatment, these genes did not show specific conservation in their coexpression network. This result indicates that conclusions on gene expression conservation can be generic (e. g., ribosomal-related genes) or true under only very specifically defined conditions.

Condition-specific LNS identified mis-annotated genes in $S$. bayanus that were overlooked by the global LNS analysis. For example, S. bayanus gene 636.13 was matched to CLB2 (YPR119W) in the initial annotation efforts by [8]. However, it has a low cell cycle LNS $(-0.03)$ and this lack of expression conservation is evident by its shift in peak expression during the cell cycle (Additional file 3: Figure S3). 636.13 instead corresponds to CLB5 based on both synteny and Blast alignment [44]. This change in gene expression was observable in the condition-specific LNS analysis of cell cycle synchronized cells because this alternate phase of expression changed the correlations with other periodically regulated genes. In the global dataset that consisted of primarily asynchronous cells, these phase specific correlations were not present.

We take two examples here to illustrate major changes in the expression response to environmental change between the two species. First, we quantized the expression data from the diauxic shift in S. bayanus and S. cerevisiae [58] based on their diauxic shift condition-specific LNS (Figure 4A-B). We observed that although the majority of the genes preserved their expression response upon diauxic shift, the lower quartile (by condition-specific LNS) of the orthologs displayed largely anti-correlated expression. Accordingly, 941 orthologs displayed a negative condition-specific LNS in diauxic shift. S. bayanus and $S$. cerevisiae have several observed differences in fermentation behavior [60-62], some of which could be associated with the changes in gene expression that we observe. 


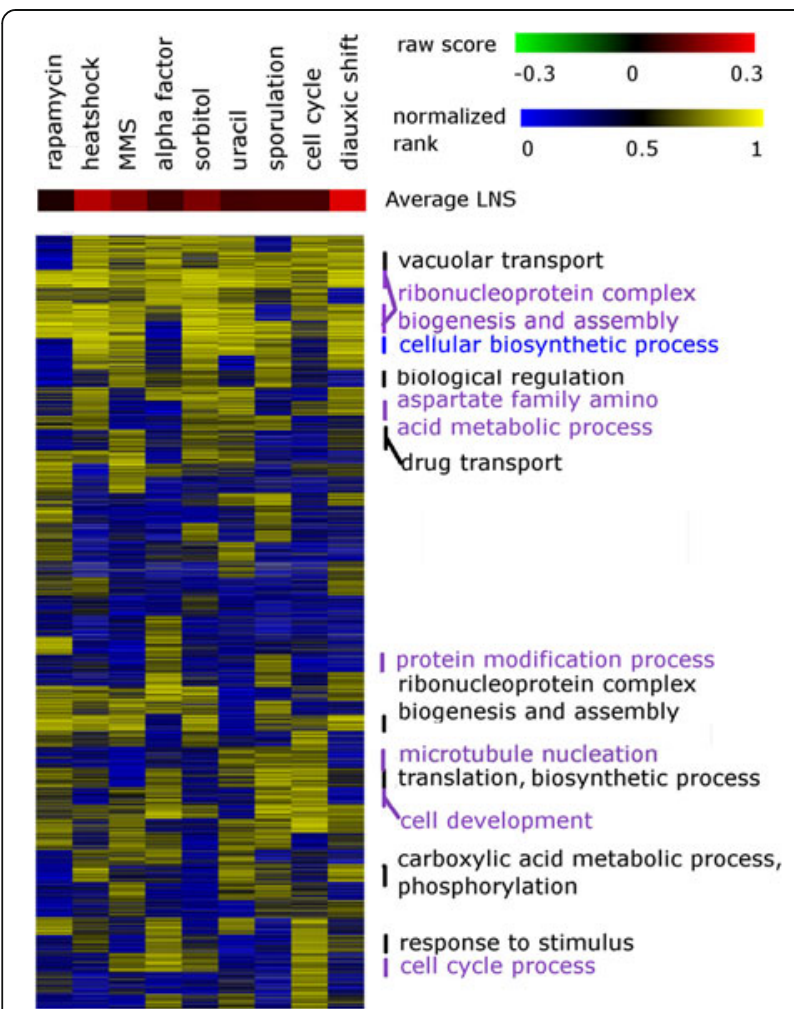

Figure 3 Variation in expression conservation of genes of different functional groups under different perturbations. The LNS for each gene for matched datasets of the two species was calculated. LNS scores were first k-means clustered and then arranged hierarchically by the centers of these clusters, and the scores presented by a heat map. GO biological process enrichment for each cluster was determined. Enriched terms with a Bonferonnicorrected $p$ value lower than 0.01 are labeled. The expression patterns of ribosome-related genes are conserved upon most perturbations. However, the expression of many of the genes of other functions is only conserved under specific conditions. The range of LNS scores varies for different datasets. Datasets with large magnitude expression changes tend to have a greater LNS range; dataset size and quality also influence the range of LNS.

A similarly large fraction of divergent genes was observed for the paired cell cycle data. We identified 591 (in S. bayanus) and 626 (in S. cerevisiae) cell cycleregulated genes whose one-to-one orthologs have data in the other species, making approximately the same proportion of genes cell cycle-regulated in both species. In total this represents 1106 unique genes, with 111 pairs of orthologous genes periodic in both $S$. bayanus and $S$. cerevisiae ( $p<0.002$, two tailed $t$-test). We also assessed the conservation of cell cycle specific gene expression using the 800 genes previously identified by a different dataset [55] as periodically expressed in synchrony with the cell cycle in S. cerevisiae, and observed that 226 of these orthologs were periodically expressed in synchronous cultures of $S$. bayanus $(\mathrm{p}<0.001)$. A large fraction of the periodically expressed genes were only cell cycle regulated in one of the species (Figure 4C-D): of the 1106 cell cycle regulated genes identified in either species, 258 had a cell cycle-specific LNS lower than 0, indicating significant change in their behavior over the cell cycle.

During the cell cycle, either phase changes or a presence of periodic expression in only one species could contribute to low LNS. We have recently correlated some of these changes in periodic gene expression with differential motif presence and nucleosome occupancy in these genes' promoters [10]. Some of the differences in timing could result from changes in the regulation of the cell cycle, but coherent cycling of protein levels could also be achieved even when gene expression is divergent. For example, if one member of a protein complex was periodically expressed in one species, another member of the same complex could be periodically expressed in the other species. This scenario would result in divergence of expression pattern even though the protein complex was periodically regulated through the availability of the cycling component $[45,63,64]$. Indeed we observe that although most cell cycle protein complexes retain cell cycle-regulated genes in both species, the identity of dynamic members differs between species (Additional file 3: Figure S4). These observations of expression divergence are not limited to the specific examples described above: all datasets have a fraction of divergently expressed genes despite the general trend of expression conservation observed over all data, underlining the importance of a dataset-specific measurement of expression conservation.

\section{Discussion}

In this study, we employed a scalable measurement of expression conservation between species, Local Network Similarity, or LNS, to study the conservation of gene expression networks using large microarray data compendia from S. bayanus and S. cerevisiae. This unsupervised metric allowed us to quantify expression divergence between orthologs for datasets that are different in timecourse sampling, or for species that have differential response kinetics to environmental perturbations. This distance metric scales the measurement of expression conservation between -1 and 1 , with the null-hypothesis distribution centered at zero. Future research directions include extension of this metric to greater evolutionary distances and diverse data types beyond gene expression. We expect that the normalization inherent in the LNS metric will make it particularly robust for RNA-seq data in the face of the larger noise component that has been observed for genes with low expression levels [65-68].

Using the newly developed LNS metric, we found that patterns of expression-level divergence vary among different biological processes and functional groups. 


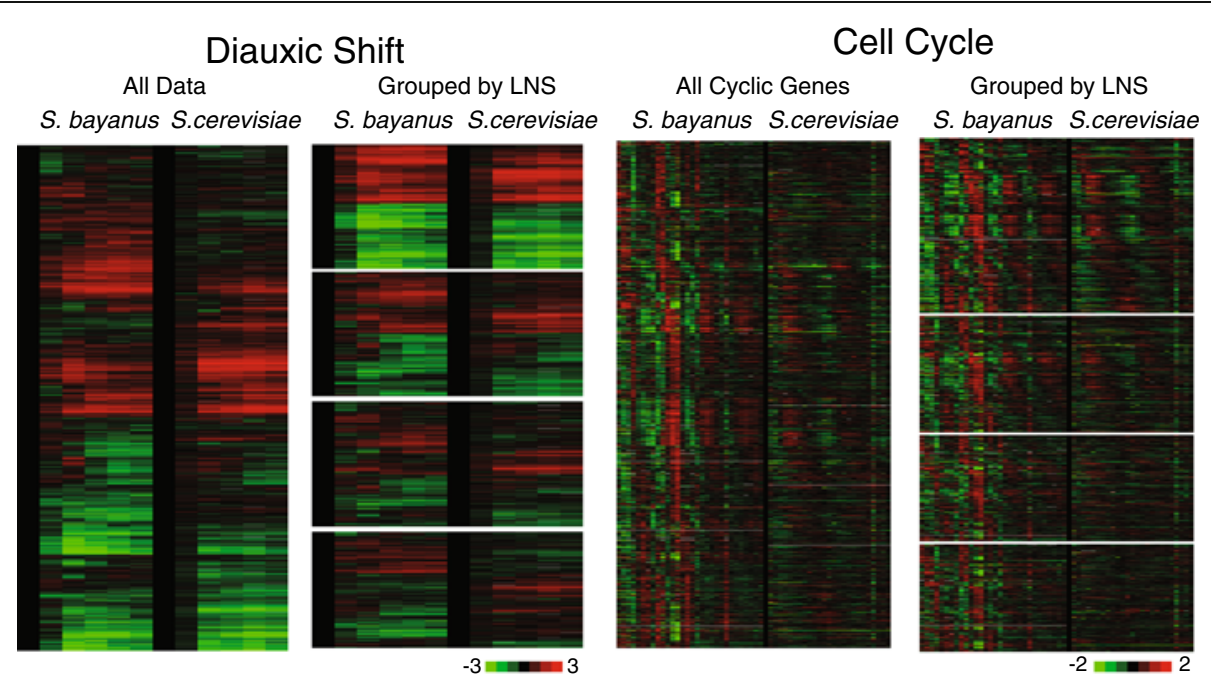

Figure 4 Condition-specific LNS sorting of expression data into conserved and non-conserved patterns. A. Expression data from the diauxic shift in both S. bayanus and S. cerevisiae were separately zero-transformed, and then aligned so that each horizontal line of data contains the expression of an orthologous pair of genes. The paired expression data were hierarchically clustered by uncentered Pearson correlation [59]. The data are presented by a heat map of $\log _{2}$ expression values. S. cerevisiae diauxic shift data were from [58]. B. The clustered data were partitioned by LNS score into quartiles, preserving the order of the genes in the original cluster. Genes with different expression between the species have low LNS scores. C. Cell cycle data from MATa cells synchronized by alpha factor arrest were mean centered. S. cerevisiae cell cycle data was obtained from [57]. For each species, the phase of the cell cycle was determined by Fourier transformation, and the top genes mapped to the phase were determined as cell cycle-regulated. Orthologs with either member of the ortholog pair determined as cell cycle-regulated are presented. The orthologs were arranged by the time of peak expression in S. bayanus.

Certain central processes such as ribosome biogenesis are highly conserved on both the sequence and expression level. However, other functional groups involved in seemingly conserved behavior (e.g. cell cycle, diauxic shift) in fact include a significant fraction of orthologs whose expression diverges. This indicates that specific expression patterning of some genes is not critical to an organism's response to environmental change. On the other hand, the overall conserved expression patterns between the two species might represent genes with key functional roles in responding to specific environmental changes.

One limitation of the current study is that we cannot determine to what degree sequence divergence explains overall expression network divergence between S. bayanus and S. cerevisiae. The selective forces acting on gene expression are as yet unclear and deriving a null model for gene expression evolution is a topic of active research (reviewed in [69-71]). Our observation of a bimodal distribution of LNS scores suggests that some genes could be evolving under selection for conserved expression patterns, while others may evolve more neutrally and thus show greater variance [72-74]. However, those genes that appear to be evolving neutrally could be simply not specifically perturbed in the conditions used for the available expression data. Addressing this challenge experimentally requires further collection of diverse expression datasets in genetically divergent strains within these species, as well as in other species across a range of evolutionary distances. Using such datasets, LNS can be used to delineate further how expression differences change with varying levels of sequence change.

\section{Conclusions}

This study focuses on the response and expression profiling of $S$. bayanus under different perturbations. Complementary studies of regulatory networks, such as interrogation of transcription factor occupancy [75] and nucleosome positioning [10,76], will be useful to more fully characterize changes between species. Furthermore, while here we provide a prototype application of our network-based divergence measure (LNS) to gene expression, this approach should be extendable to other types of genome-wide data and can encompass diverse types of quantification of co-expression and/or network similarity. Extending our comparative approach to other groups of related species, such as Candida yeasts, Drosophila species, and mammals, could extend the observations made here. Since our experimental and analytical frameworks are agnostic to species and platform, they should be transferable to other systems. Such studies can be combined with sequence analysis to yield a more complete understanding of the mode of phenotype evolution and its relationship with sequence changes. 


\section{Methods}

Pre-processing of S. cerevisiae and S. bayanus data

We assembled 303 arrays covering 46 datasets in $S$. bayanus [GEO: GSE16544] and 2569 arrays covering 125 datasets in $S$. cerevisiae. To allow reasonable comparison between datasets, we performed the following normalization steps. For each dataset, genes that are represented in less than $50 \%$ of the arrays were removed from this dataset, and missing values were estimated using KNNimpute with $\mathrm{K}=10$, Euclidean distance [77]. Finally, biological replicates are averaged, resulting in datasets with each gene followed by a vector representing its expression values in a series of arrays.

\section{Calculation of pair-wise correlation}

For each dataset $i$, between gene pair expression vectors $j$ and $k$, we calculated the correlation coefficient of their expression pattern:

$$
\rho\left(j_{i}, k_{i}\right)=\frac{\operatorname{cov}\left(j_{i}, k_{i}\right)}{\sigma_{j_{i}} \sigma_{k_{i}}}
$$

To allow comparison between datasets, we Fisher's ztransformed these correlation values [78]:

$$
z\left(j_{i}, k_{i}\right)=\frac{1}{2} \ln \frac{1+\rho\left(j_{i}, k_{i}\right)}{1-\rho\left(j_{i}, k_{i}\right)}
$$

For each dataset, these $\mathrm{z}$ values were then normalized to $Z$ ' $N(0,1)$. We define the connection weights $z(j, k)$ between any two genes $j$ and $k$ in a species as the average of the normalized $z$ values over all datasets. This forms a pair-wise connection weight matrix for each species.

\section{Calculation of local network similarity (LNS) as a measurement of expression divergence}

Connection weights between a specific gene $j$ to all others form a vector $W(j)=(z(1, j), z(2, j) \ldots z(N, j))$, where $\mathrm{N}$ is the total number of matched orthologs. LNSj,j' is defined as the correlation between the matched vectors (by orthology) of the two species, quantifying the conservation of the overall expression pattern of gene $j$ (with its ortholog being j') (Figure 1):

$$
L N S_{j, j^{\prime}}=\frac{\operatorname{cov}\left(W(j), W\left(j^{\prime}\right)\right)}{\sigma_{W(j)} \sigma_{W\left(j^{\prime}\right)}}
$$

To assess the conservation level of orthologs upon specific biological perturbation (condition-specific LNS), we manually selected 10 datasets in S. bayanus that have matched time-course data in $S$. cerevisiae. For each data pair, we define the connection weight as the standard normalized value of formula (2), followed by the calculation of condition-specific LNS according to formula (3) (Figure 3).

\section{Correlation between sequence divergence and LNS}

Measures of sequence divergence were used as in [26], including $\mathrm{dN}, \mathrm{dN} / \mathrm{dS}, \mathrm{Ka}$, and $\mathrm{Ka} / \mathrm{Ks}$ as previously calculated $[7,79]$. A normal distribution was obtained by $\log _{2}$ transforming the data, mean subtracting it, and normalizing by the standard deviation. Correlation was calculated using the Pearson correlation.

\section{Clustering of condition-specific LNS and functional enrichment analysis}

We determined the number of clusters in the conditionspecific LNS matrix according to [80], resulting in 48 clusters. The enrichment of genes in each cluster was calculated through the program GOTermFinder [81].

\section{Identification of cell cycle regulated expression}

We acquired S. cerevisiae cell cycle data from [57]. The following steps of identification of cell cycle regulated genes were applied to each species. For each gene in the cell cycle data, the expression values were centered so that the average over the time course equals to 0. A Fourier transformation was applied to the dataset of individual species so as to identify the period of cell cycle [55]. In $S$. bayanus the top 613 genes mapping to the phase were chosen as cell cycle regulated; and 785 genes in $S$. cerevisiae were chosen. This corresponds to 601 and 644 genes in S. bayanus and S. cerevisiae having one-to-one orthologs in the other species respectively. Among them, 591 (S. bayanus) and 626 (S. cerevisiae) have expression data in the other species, with 111 pairs overlapping.

\section{Additional files}

Additional file 1: Table S1. Global between-species LNS for S. cerevisiae and S. bayanus. Table S1 presents the global LNS for all ortholog pairs in S. cerevisiae and S. bayanus.

Additional file 2: Table S2. Within-species LNS for S. cerevisiae. Table S2 presents the LNS calculated within S. cerevisiae by subsampling the dataset.

Additional file 3: Figure S1. Correlation of LNS with sequence features. The $r$ value calculated by Pearson correlation between the LNS and the conservation of the indicated sequence is indicated. The p-value is calculated by using a test randomizing sequence similarity and LNS matches. Because the presence and conservation of a TATA box is a categorical value, that correlation is calculated using the point-biserial correlation. Figure S2. Correlation of LNS and various measures of sequence divergence. Expression similarity between orthologs, quantified as local network similarity (LNS), versus sequence similarity. LNS is correlated with indicated sequence divergence metrics with Pearson correlations of $-0.215(p<2.2 e-16)$ for $d N / d S,-0.238(p<2.2 e-16)$ for adjusted dN/dS, -0.222 ( $p<2.2 \mathrm{e}-16)$ for Ka, and -0.169 ( $p=1.70 \mathrm{e}-14)$ for $\mathrm{Ks} / \mathrm{Ks}$. Black line is loess smoothed curve line and red line is running average of LNS summed over 100-gene windows. Figure S3. Cell-cyclespecific LNS identifies mis-annotation of S. bayanus gene 636.13. Among the central cell cycle-regulated genes, CLB2 (636.13) had a particularly low condition-specific LNS (-0.03). The expression pattern of this gene was shifted during the cell cycle. Analysis of sequence and synteny reveals 
that 636.13 is the ortholog of CLB5 rather than CLB2. Figure S4.

Orthologous complexes involving cell-cycle regulated genes. S. cerevisiae MIPS protein complexes curated by (de Lichtenberg, Jensen et al. 2005) with more than 4 orthologous genes were included if at least one of the genes is cell-cycle regulated (as determined in the main text) in either species. Nodes depict genes and lines connecting them depict physical interactions. Cell cycle regulated genes are highlighted in red: A. S. bayanus B. S. cerevisiae. For complexes that have cell cycle regulated genes in both species, the dynamic member frequently switches.

Additional file 4: Table S3. Condition-specific between-species LNS for S. cerevisiae and S. bayanus. Table S3. presents the condition-specific LNS for each gene.

\section{Competing interests}

The authors declare they have no competing interests.

\section{Authors' contributions}

YF developed the LNS metric, calculated global LNS, and correlated it to sequence features. MJD and AAC analyzed LNS data and sequence similarity measures. YF calculated and clustered condition-specific LNS. All authors conceived the study, participated in interpretation of the results, and wrote the paper. All authors read and approved the final manuscript.

\section{Acknowledgements}

Thanks to Greg Lang for GPA1 data in advance of publication; David Botstein, Ian Ehrenreich, Justin Gerke, Greg Lang, and Dannie Durand for helpful comments; and Kevin Byrne for orthology mapping data. OGT is supported by the NIH grant R01 GM071966, NSF grant IIS-0513552 and NSF CAREER award DBI-0546275. All authors were supported by the NIGMS Center of Excellence P50 GM071508, and by donations from the A. V. Davis Foundation and Princeton University for funding of QCB301, Experimental Project Laboratory. MJD is supported in part by grants from the National Center for Research Resources (5P41RR011823-17) and the National Institute of General Medical Sciences (8 P41 GM103533-17) from the National Institutes of Health. MJD is a Rita Allen Foundation Scholar. OGT is supported by the NIH grants R01 GM071966 and R01HG005998, NSF grant IIS-0513552 and NSF CAREER award DBI-0546275. AAC is supported by the Canadian Institutes for Health Research.

\section{Author details}

'Lewis-Sigler Institute for Integrative Genomics, Princeton University, Princeton, NJ 08544, USA. ${ }^{2}$ Department of Molecular Biology, Princeton University, Princeton, NJ 08544, USA. ${ }^{3}$ Current Address: Department of Computational Medicine \& Bioinformatics, University of Michigan, Ann Arbor, MI 48109, USA. ${ }^{4}$ Department of Genome Sciences, University of Washington, Seattle, WA 98195, USA. ${ }^{5}$ Department of Computer Science, Princeton University, Princeton, NJ 08540, USA. ${ }^{6}$ Donnelly Centre for Cellular and Biomolecular Research, University of Toronto, Toronto, Ontario M5S 3E1, Canada.

Received: 8 October 2012 Accepted: 3 January 2013

Published: 16 January 2013

\section{References}

1. Galagan JE, Henn MR, Ma L, Cuomo CA, Birren B: Genomics of the fungal kingdom: insights into eukaryotic biology. Genome Res 2005, 15(12):1620-1631.

2. Kellis M, Birren BW, Lander ES: Proof and evolutionary analysis of ancient genome duplication in the yeast Saccharomyces cerevisiae. Nature 2004, 428(6983):617-624.

3. Dietrich FS, Voegeli S, Brachat S, Lerch A, Gates K, Steiner S, Mohr C, Pohlmann R, Luedi P, Choi S, et al: The Ashbya gossypii genome as a tool for mapping the ancient Saccharomyces cerevisiae genome. Science 2004, 304(5668):304-307.

4. Wapinski I, Pfeffer A, Friedman N, Regev A: Natural history and evolutionary principles of gene duplication in fungi. Nature 2007 449(7158):54-61.

5. Scannell DR, Frank AC, Conant GC, Byrne KP, Woolfit M, Wolfe KH: Independent sorting-out of thousands of duplicated gene pairs in two yeast species descended from a whole-genome duplication. Proc Natl Acad Sci USA 2007, 104(20):8397-8402.
6. Dujon B, Sherman D, Fischer G, Durrens P, Casaregola S, Lafontaine I, De Montigny J, Marck C, Neuveglise C, Talla E, et al: Genome evolution in yeasts. Nature 2004, 430(6995):35-44.

7. Kellis M, Patterson N, Endrizzi M, Birren B, Lander ES: Sequencing and comparison of yeast species to identify genes and regulatory elements. Nature 2003, 423(6937):241-254.

8. Cliften P, Sudarsanam P, Desikan A, Fulton L, Fulton B, Majors J, Waterston $\mathrm{R}$, Cohen BA, Johnston M: Finding functional features in Saccharomyces genomes by phylogenetic footprinting. Science 2003, 301(5629):71-76.

9. Gasch AP, Moses AM, Chiang DY, Fraser HB, Berardini M, Eisen MB: Conservation and evolution of cis-regulatory systems in ascomycete fungi. PLOS Biol 2004, 2(12):e398.

10. Guan Y, Yao V, Tsui K, Gebbia M, Dunham MJ, Nislow C, Troyanskaya OG: Nucleosome-coupled expression differences in closely-related species. BMC Genomics 2011, 12:466.

11. Tsong AE, Miller MG, Raisner RM, Johnson AD: Evolution of a combinatorial transcriptional circuit: a case study in yeasts. Cell 2003, 115(4):389-399.

12. Tsong AE, Tuch BB, Li H, Johnson AD: Evolution of alternative transcriptional circuits with identical logic. Nature 2006, 443(7110):415-420.

13. Ihmels J, Bergmann S, Berman J, Barkai N: Comparative gene expression analysis by differential clustering approach: application to the Candida albicans transcription program. PLoS Genet 2005, 1(3):e39.

14. Gasch AP: Comparative genomics of the environmental stress response in ascomycete fungi. Yeast 2007, 24(11):961-976.

15. Rustici G, van Bakel H, Lackner DH, Holstege FC, Wijmenga C, Bahler J, Brazma A: Global transcriptional responses of fission and budding yeast to changes in copper and iron levels: a comparative study. Genome Biol 2007, 8(5):R73.

16. Wohlbach DJ, Kuo A, Sato TK, Potts KM, Salamov AA, Labutti KM, Sun H, Clum A, Pangilinan JL, Lindquist EA, et al: Comparative genomics of xylose-fermenting fungi for enhanced biofuel production. Proc Natl Acad Sci 2011, 108(32):13212-13217.

17. Scannell DR, Zill OA, Rokas A, Payen C, Dunham MJ, Eisen MB, Rine J, Johnston M, Hittinger CT: The Awesome Power of Yeast Evolutionary Genetics: New Genome Sequences and Strain Resources for the Saccharomyces sensu stricto Genus. G3 (Bethesda, Md) 2011, 1(1):11-25.

18. Liti G, Carter DM, Moses AM, Warringer J, Parts L, James SA, Davey RP, Roberts IN, Burt A, Koufopanou V, et al: Population genomics of domestic and wild yeasts. Nature 2009, 458(7236):337-341.

19. Bullard JH, Mostovoy Y, Dudoit S, Brem RB: Polygenic and directional regulatory evolution across pathways in Saccharomyces. Proc Natl Acad Sci 2010, 107(11):5058-5063.

20. Horinouchi T, Yoshikawa K, Kawaide R: Genome-wide expression analysis of Saccharomyces pastorianus orthologous genes using oligonucleotide microarrays. J Biosci Bioeng 2010, 110(5):602-607.

21. Martin OC, DeSevo CG, Guo BZ, Koshland DE, Dunham MJ, Zheng Y: Telomere behavior in a hybrid yeast. Cell Res 2009, 19(7):910.

22. Tirosh I, Reikhav S, Levy AA, Barkai N: A yeast hybrid provides insight into the evolution of gene expression regulation. Science (New York, NY) 2009, 324(5927):659-662.

23. Zill OA, Scannell D, Teytelman L, Rine J: Co-evolution of transcriptional silencing proteins and the DNA elements specifying their assembly. PLOS Biol 2010, 8(11):e1000550.

24. Tirosh I, Weinberger A, Carmi M, Barkai N: A genetic signature of interspecies variations in gene expression. Nat Genet 2006, 38(7):830-834

25. Tirosh I, Weinberger A, Bezalel D, Kaganovich M, Barkai N: On the relation between promoter divergence and gene expression evolution. Mol Syst Biol 2008, 4:159

26. Tirosh I, Barkai N: Evolution of gene sequence and gene expression are not correlated in yeast. Trends Genet 2008, 24(3):109-113.

27. Lelandais G, Tanty V, Geneix C, Etchebest C, Jacq C, Devaux F: Genome adaptation to chemical stress: clues from comparative transcriptomics in Saccharomyces cerevisiae and Candida glabrata. Genome Biol 2008, 9(11):R164.

28. Guan Y, Dunham M, Caudy A, Troyanskaya O: Systematic planning of genome-scale experiments in poorly studied species. PLoS Comput Biol 2010, 6(3):e1000698.

29. Flintoft L: Learning to prioritize. Nat Rev Genet 2010, 11(5):315.

30. Parkinson H, Kapushesky M, Shojatalab M, Abeygunawardena N, Coulson R, Farne A, Holloway E, Kolesnykov N, Lilja P, Lukk M, et al: ArrayExpress-a public database of microarray experiments and gene expression profiles. Nucleic Acids Res 2007, 35(Database issue):D747-D750. 
31. Ball CA, Jin H, Sherlock G, Weng S, Matese JC, Andrada R, Binkley G, Dolinski K, Dwight SS, Harris MA, et al: Saccharomyces Genome Database provides tools to survey gene expression and functional analysis data. Nucleic Acids Res 2001, 29(1):80-81.

32. Edgar R, Domrachev M, Lash AE: Gene Expression Omnibus: NCBI gene expression and hybridization array data repository. Nucleic Acids Res 2002, 30(1):207-210.

33. Le Crom S, Devaux F, Jacq C, Marc P: yMGV: helping biologists with yeast microarray data mining. Nucleic Acids Res 2002, 30(1):76-79.

34. Marinelli RJ, Montgomery K, Liu CL, Shah NH, Prapong W, Nitzberg M, Zachariah ZK, Sherlock GJ, Natkunam Y, West RB, et al: The Stanford Tissue Microarray Database. Nucleic Acids Res 2008, 36(Database issue):D871-D877.

35. Dutilh BE, Huynen MA, Snel B: A global definition of expression context is conserved between orthologs, but does not correlate with sequence conservation. BMC Genomics 2006, 7:10

36. Tirosh I, Barkai N: Comparative analysis indicates regulatory neofunctionalization of yeast duplicates. Genome Biol 2007, 8(4):R50.

37. Brem RB, Yvert G, Clinton R, Kruglyak L: Genetic dissection of transcriptional regulation in budding yeast. Science (New York, NY) 2002, 296(5568):752-755.

38. Fay JC, McCullough HL, Sniegowski PD, Eisen MB: Population genetic variation in gene expression is associated with phenotypic variation in Saccharomyces cerevisiae. Genome Biol 2004, 5(4):R26.

39. Smith EN, Kruglyak L: Gene-environment interaction in yeast gene expression. PLoS Biol 2008, 6(4):e83.

40. Knight SA, Labbe S, Kwon LF, Kosman DJ, Thiele DJ: A widespread transposable element masks expression of a yeast copper transport gene. Genes Dev 1996, 10(15):1917-1929.

41. Lang Gl, Murray AW, Botstein D: The cost of gene expression underlies a fitness trade-off in yeast. Proc Natl Acad Sci USA 2009, 106(14):5755-5760.

42. Yvert G, Brem RB, Whittle J, Akey JM, Foss E, Smith EN, Mackelprang R, Kruglyak L: Trans-acting regulatory variation in Saccharomyces cerevisiae and the role of transcription factors. Nat Genet 2003, 35(1):57-64.

43. Zill OA, Rine J: Interspecies variation reveals a conserved repressor of alphaspecific genes in Saccharomyces yeasts. Genes Dev 2008, 22(12):1704-1716.

44. Gordon JL, Byrne KP, Wolfe KH: Additions, losses, and rearrangements on the evolutionary route from a reconstructed ancestor to the modern Saccharomyces cerevisiae genome. PLoS Genet 2009, 5(5):e1000485.

45. Jordan IK, Marino-Ramirez L, Koonin EV: Evolutionary significance of gene expression divergence. Gene 2005, 345(1):119-126.

46. Khaitovich P, Hellmann I, Enard W, Nowick K, Leinweber M, Franz H, Weiss G, Lachmann M, Paabo S: Parallel patterns of evolution in the genomes and transcriptomes of humans and chimpanzees. Science 2005, 309 (5742):1850-1854.

47. Liao BY, Zhang J: Evolutionary conservation of expression profiles between human and mouse orthologous genes. Mol Biol Evol 2006, 23(3):530-540.

48. Sartor MA, Zorn AM, Schwanekamp JA, Halbleib D, Karyala S, Howell ML, Dean GE, Medvedovic M, Tomlinson CR: A new method to remove hybridization bias for interspecies comparison of global gene expression profiles uncovers an association between mRNA sequence divergence and differential gene expression in Xenopus. Nucleic Acids Res 2006, 34(1):185-200.

49. Nuzhdin SV, Wayne ML, Harmon KL, Mclntyre LM: Common pattern of evolution of gene expression level and protein sequence in Drosophila. Mol Biol Evol 2004, 21(7):1308-1317.

50. Lemos B, Bettencourt BR, Meiklejohn CD, Hartl DL: Evolution of proteins and gene expression levels are coupled in Drosophila and are independently associated with mRNA abundance, protein length, and number of proteinprotein interactions. Mol Biol Evol 2005, 22(5):1345-1354.

51. Shalem O, Dahan O, Levo M, Martinez MR, Furman I, Segal E, Pilpel Y: Transient transcriptional responses to stress are generated by opposing effects of mRNA production and degradation. Mol Syst Biol 2008, 4:223.

52. Gasch AP, Spellman PT, Kao CM, Carmel-Harel O, Eisen MB, Storz G, Botstein D, Brown PO: Genomic expression programs in the response of yeast cells to environmental changes. Mol Biol Cell 2000, 11(12):4241-4257.

53. Saldanha AJ, Brauer MJ, Botstein D: Nutritional homeostasis in batch and steady-state culture of yeast. Mol Biol Cell 2004, 15(9):4089-4104.

54. Urban J, Soulard A, Huber A, Lippman S, Mukhopadhyay D, Deloche O, Wanke V, Anrather D, Ammerer G, Riezman H, et al: Sch9 is a major target of TORC1 in Saccharomyces cerevisiae. Mol Cell 2007, 26(5):663-674.
55. Spellman PT, Sherlock G, Zhang MQ, lyer VR, Anders K, Eisen MB, Brown PO, Botstein D, Futcher B: Comprehensive identification of cell cycleregulated genes of the yeast Saccharomyces cerevisiae by microarray hybridization. Mol Biol Cell 1998, 9(12):3273-3297.

56. Primig M, Williams RM, Winzeler EA, Tevzadze GG, Conway AR, Hwang SY, Davis RW, Esposito RE: The core meiotic transcriptome in budding yeasts. Nat Genet 2000, 26(4):415-423.

57. Pramila T, Wu W, Miles S, Noble WS, Breeden LL: The Forkhead transcription factor $\mathrm{Hcm} 1$ regulates chromosome segregation genes and fills the S-phase gap in the transcriptional circuitry of the cell cycle. Genes Dev 2006, 20(16):2266-2278.

58. Brauer MJ, Saldanha AJ, Dolinski K, Botstein D: Homeostatic adjustment and metabolic remodeling in glucose-limited yeast cultures. Mol Biol Cell 2005, 16(5):2503-2517

59. Eisen MB, Spellman PT, Brown PO, Botstein D: Cluster analysis and display of genome-wide expression patterns. Proc Natl Acad Sci USA 1998, 95 (25):14863-14868

60. Arroyo-López FN, Salvadó Z, Tronchoni J, Guillamón JM, Barrio E, Querol A: Susceptibility and resistance to ethanol in Saccharomyces strains isolated from wild and fermentative environments. Yeast (Chichester, England) 2010, 27(12):1005-1015.

61. Masneuf-Pomarède I, Bely M, Marullo P, Lonvaud-Funel A, Dubourdieu D: Reassessment of phenotypic traits for Saccharomyces bayanus var. uvarum wine yeast strains. Int J Food Microbiol 2010, 139(1-2):79-86.

62. Salvadó Z, Arroyo-López FN, Guillamón JM, Salazar G, Querol A, Barrio E: Temperature adaptation markedly determines evolution within the genus Saccharomyces. Appl Environ Microbiol 2011, 77(7):2292-2302.

63. de Lichtenberg $U$, Jensen $L$, Brunak S, Bork P: Dynamic complex formation during the yeast cell cycle. Science 2005, 307(5710):724-727.

64. Jensen $\sqcup$, Jensen TS, de Lichtenberg U, Brunak S, Bork P: Co-evolution of transcriptional and post-translational cell-cycle regulation. Nature 2006, 443(7111):594-597.

65. Tarazona S, Garcia-Alcalde F, Dopazo J, Ferrer A, Conesa A: Differential expression in RNA-seq: A matter of depth. Genome Res 2011, 21(12):2213-2223.

66. McIntyre LM, Lopiano KK, Morse AM, Amin V, Oberg AL, Young LJ, Nuzhdin SV: RNA-seq: technical variability and sampling. BMC Genomics 2011, 12(1):293.

67. Liu S, Lin L, Jiang P, Wang D, Xing Y: A comparison of RNA-Seq and highdensity exon array for detecting differential gene expression between closely related species. Nucleic Acids Res 2011, 39(2):578-588.

68. Bloom JS, Khan Z, Kruglyak L, Singh M, Caudy AA: Measuring differential gene expression by short read sequencing: quantitative comparison to 2-channel gene expression microarrays. BMC Genomics 2009, 10:221.

69. Gilad Y, Oshlack A, Rifkin SA: Natural selection on gene expression. Trends Genet 2006, 22(8):456-461.

70. Zheng W, Gianoulis TA, Karczewski K, Zhao H, Snyder M: Regulatory variation within and between species. Annu Rev Genomics Hum Genet 2011, 12:327-346.

71. Romero IG, Ruvinsky I, Gilad Y: Comparative studies of gene expression and the evolution of gene regulation. Nat Rev Genet 2012, 13(7):505-516.

72. Whitehead A, Crawford DL: Neutral and adaptive variation in gene expression. Proc Natl Acad Sci USA 2006, 103(14):5425-5430.

73. Rifkin SA, Kim J, White KP: Evolution of gene expression in the Drosophila melanogaster subgroup. Nat Genet 2003, 33(2):138-144.

74. Khaitovich P, Pääbo S, Weiss G: Toward a neutral evolutionary model of gene expression. Genetics 2005, 170(2):929-939.

75. Borneman AR, Gianoulis TA, Zhang ZD, Yu H, Rozowsky J, Seringhaus MR, Wang LY, Gerstein M, Snyder M: Divergence of transcription factor binding sites across related yeast species. Science 2007, 317(5839):815-819.

76. Tsankov AM, Thompson DA, Socha A, Regev A, Rando OJ: The role of nucleosome positioning in the evolution of gene regulation. PLOS Biol 2010, 8(7):e1000414.

77. Troyanskaya O, Cantor M, Sherlock G, Brown P, Hastie T, Tibshirani R, Botstein D, Altman RB: Missing value estimation methods for DNA microarrays. Bioinformatics (Oxford, England) 2001, 17(6):520-525.

78. Fisher RA: Frequency distribution of the values of the correlation coefficients in samples from an indefinitely large population. Biometrika 1915, 10(4):507-521.

79. Wall DP, Hirsh AE, Fraser HB, Kumm J, Giaever G, Eisen MB, Feldman MW: Functional genomic analysis of the rates of protein evolution. Proc Natl Acad Sci USA 2005, 102(15):5483-5488. 
80. Mardia KV, Kent T, Bibby JM: Multivariate Analysis. sixthth edition. London, New York, Toronto, Sydney, San Francisco: Academic Press; 1997.

81. Boyle El, Weng S, Gollub J, Jin H, Botstein D, Cherry JM, Sherlock G: GO:TermFinder-open source software for accessing Gene Ontology information and finding significantly enriched Gene Ontology terms associated with a list of genes. Bioinformatics 2004, 20(18):3710-3715.

doi:10.1186/1471-2164-14-33

Cite this article as: Guan et al:: Comparative gene expression between two yeast species. BMC Genomics 2013 14:33.

\section{Submit your next manuscript to BioMed Central} and take full advantage of:

- Convenient online submission

- Thorough peer review

- No space constraints or color figure charges

- Immediate publication on acceptance

- Inclusion in PubMed, CAS, Scopus and Google Scholar

- Research which is freely available for redistribution 